\title{
Seleção de herbicidas para o controle de plantas voluntárias de soja resistentes ao glyphosate ${ }^{1}$
}

\author{
Herbicides selection for controlling soybean volunteer plants resistant to glyphosate
}

\author{
Dayene Bueno Cruvinel Lima ${ }^{2 *}$, Alessandro Guerra da Silva $^{3}$, Sérgio de Oliveira Procópio ${ }^{3}$, \\ Alberto Leão de Lemos Barroso ${ }^{3}$, Hugo de Almeida Dan ${ }^{4}$, Eduardo Barbosa Costa ${ }^{5}$, \\ Antonio Joaquim Braga Pereira ${ }^{3}$
}

\begin{abstract}
Resumo - Este trabalho objetivou avaliar a eficácia de herbicidas no controle de plantas voluntárias de soja resistente ao glyphosate. O ensaio foi conduzido a campo utilizando o delineamento de blocos casualizados, em esquema fatorial $6 \times 9$, com quatro repetições, sendo o primeiro fator seis variedades de soja resistentes ao herbicida glyphosate (BRS Favorita RR ${ }^{\circledR}$, BRS Valiosa RR ${ }^{\circledR}$, CD 219 RR $^{\circledR}$, M-SOY 7908 RR $^{\circledR}$, P98Y11 ${ }^{\circledR}$ e P98R31 ${ }^{\circledR}$ ) e o segundo herbicidas aplicados isolados ou em mistura (2,4-D, 2,4-D + glyphosate, metsulfuron-methyl, metsulfuron-metyl + glyphosate, [MSMA + diuron], [MSMA + diuron] + glyphosate, [paraquat + diuron], [paraquat + diuron] + glyphosate e uma testemunha sem herbicida). Os tratamentos [MSMA + diuron] e [MSMA + diuron] + glyphosate apresentaram controle eficaz apenas para CD 219 RR $^{\circledR}$. Metsulfuron-methyl + glyphosate apresentou eficácia no controle da M-SOY 7908 $\mathrm{RR}^{\circledR}, \mathrm{P} 98 \mathrm{Y} 11^{\circledR}, \mathrm{P} 98 \mathrm{R} 31^{\circledR}$, BRS Favorita $\mathrm{RR}^{\circledR}$ e CD $219 \mathrm{RR}^{\circledR}$; assim como a cultivar BRS Valiosa $R^{\circledR}$ foi a mais tolerante a esse tratamento. A adição de glyphosate não acarretou efeitos antagônicos na eficácia dos herbicidas, porém potencializou a ação do metsulfuron-methyl. Os herbicidas [paraquat + diuron] e 2,4-D foram eficazes no controle da soja RR. Houve diferenças na sensibilidade das variedades aos herbicidas metsulfuron-methyl, metsulfuron-methyl + glyphosate, [MSMA + diuron] e [MSMA + diuron] + glyphosate.
\end{abstract}

Palavras chaves: Glycine max; soja transgênica; Roundup Ready ${ }^{\circledR}$.

\footnotetext{
Abstract - This study aimed to evaluate the effectiveness of herbicides in controlling soybean volunteer plants resistant to glyphosate. The experiment was conducted in field conditions by using randomized complete block design in factorial scheme $6 \times 9$, with four replications, with the first factor represented by six soybean genotypes resistant to glyphosate herbicide (BRS Favorita $\mathrm{RR}^{\circledR}$, BRS Valiosa RR ${ }^{\circledR}, \mathrm{CD} 219 \mathrm{RR}^{\circledR}, \mathrm{M}-\mathrm{SOY} 7908 \mathrm{RR}^{\circledR}, \mathrm{P}^{\circledR} 8 \mathrm{Y} 11^{\circledR}$ and P98R31 ${ }^{\circledR}$ ) and the

* Autor para correspondência

${ }^{1}$ Recebido para publicação em 28/02/2011 e aceito10/04/2011.

2 Discente do Programa de Pós-graduação em Produção Vegetal da Fesurv-Universidade de Rio Verde. Email: dayenebio@hotmail.com;

${ }^{3}$ Docente do Programa de Pós-graduação em Produção Vegetal da Fesurv-Universidade de Rio Verde, Campus Universitário Fazenda Fontes do Saber, Caixa Postal 104, 75901-970, Rio Verde-GO. Email: silvaag@yahoo.com.br, procopio.so@gmail.com, all_barroso@hotmail.com,ajbpbraz@ibest.com.br;

${ }^{4}$ Discente do Programa de Pós-graduação em Agronomia da UEM;

${ }^{5}$ Discente de Agronomia da Fesurv-Universidade de Rio Verde;
}




second factor represented by herbicides sprayed isolated or in mixture (2,4-D, 2,4-D + glyphosate, metsulfuron-methyl, metsulfuron-methyl + glyphosate, [MSMA + diuron], [MSMA + diuron] + glyphosate, [paraquat + diuron], [paraquat + diuron] + glyphosate, and a check without herbicide). MSMA+diuron and [MSMA+diuron] + glyphosate treatments showed effective control only for CD $219 \mathrm{RR}^{\circledR}$. Metsulfuron-methyl+glyphosate showed efficacy in controlling M-SOY $7908 \mathrm{RR}^{\circledR}$, P98Y11 ${ }^{\circledR}, \mathrm{P} 98 \mathrm{R} 31^{\circledR}$, BRS Favorita RR ${ }^{\circledR}$ and CD $219 \mathrm{RR}^{\circledR}$; as soon as BRS Valiosa RR ${ }^{\circledR}$ was the most tolerant to this treatment. Glyphosate addition did not cause any antagonist effect in effectiveness of the herbicides, but increased the action of metsulfuron-methyl. The herbicides [Paraquat + diuron] and 2,4-D were effective in controlling RR soybean crop. There were differences in sensitivity of genotypes to metsulfuron-methyl, metsulfuron-methyl + glyphosate, [MSMA + diuron] and [MSMA + diuron] + glyphosate herbicides.

Key words: Glycine max; transgenic soybean; Roundup Ready ${ }^{\circledR}$.

\section{Introdução}

O manejo de plantas daninhas é um desafio constante para os agricultores e as inovações contínuas nessa área são essenciais para manter a efetividade dessa prática. A tecnologia que proporcionou maior impacto no manejo de plantas daninhas, nos últimos dez anos, foi a introdução de variedades tolerantes a herbicidas. A área mundial cultivada com plantas geneticamente modificadas vem aumentando nos últimos anos, com destaque para a soja (Glycine max) com resistência ao herbicida glyphosate (Roundup Ready ${ }^{\circledR}$ ), sendo a mais explorada mundialmente (Monquero, 2005).

Entre as principais vantagens da soja resistente ao glyphosate destacam-se a redução dos custos de produção (Menegatti \& Barros, 2007) e a maior flexibilidade no manejo de plantas daninhas (Webster et al., 1999). O glyphosate, herbicida não seletivo e amplamente usado em todo mundo, inibe a enzima 5-enolpiruvilchiquimato 3-fosfato sintase (EPSPs) que atua na rota biossintética de aminoácidos aromáticos (Pline-Srnic, 2006). A soja geneticamente modificada é imune aos efeitos destrutivos e letais desse herbicida e, mesmo após a sua aplicação as plantas de soja RR continuam produzindo aminoácidos aromáticos (fenilalanina, tirosina e triptofano), essenciais ao seu crescimento e desenvolvimento (Bradshaw et al., 1997). Em áreas com infestação de plantas de difícil controle, o uso de glyphosate tem se mostrado como uma ferramenta de extrema importância no manejo destas espécies (Bryson et al., 2003; Edenfield et al., 2005).

Posteriormente ao aparecimento da ferrugem asiática da soja e com a regulamentação do vazio sanitário para o Estado de Goiás, com início em 01 de julho até 30 de setembro de cada ano, o manejo químico pós-colheita das plantas voluntárias obteve grande importância no sistema de produção. Essa normatização foi implantada tendo em vista a evolução da incidência da ferrugem asiática nas lavouras, pois as plantas de soja presentes na entressafra podem servir de hospedeiras do inóculo, propiciando a proliferação do fungo causador desta doença (Terasawa et al., 2009).

A adoção do vazio sanitário já vem sendo realizada pelos agricultores, no intuito de controlar as plantas voluntárias de soja que se estabelecem após colheita. No entanto, como em cultivos convencionais o glyphosate é usado em dessecações de plantas voluntárias e plantas daninhas após a colheita da cultura. 
Em soja RR, essa prática torna-se não mais efetiva no sentido de controlar as plantas voluntárias de soja RR.

A partir desse novo cenário, é necessária a identificação de herbicidas efetivos para o controle das plantas voluntárias de soja RR e que ao mesmo tempo apresentem compatibilidade para serem utilizados em mistura com glyphosate. Desta forma, objetivou-se com o presente estudo avaliar a eficácia de herbicidas no controle de plantas voluntárias de seis variedades de soja Roundup Ready $^{\circledR}$, verificando a compatibilidade das misturas dos mesmos com glyphosate.

\section{Material e Métodos}

O ensaio foi conduzido em campo no município de Rio Verde-GO (17\%47'24', S; 5056'31' 'W; a $740 \mathrm{~m}$ de altitude). O solo da área experimental foi identificado como Latossolo Vermelho Distrófico, com textura média.

$\mathrm{O}$ delineamento experimental utilizado foi o de blocos casualizados com quatro repetições, organizado em esquema fatorial 6x9, sendo o primeiro fator constituído por seis variedades de soja resistentes ao herbicida glyphosate (BRS Favorita RR ${ }^{\circledR}$, BRS Valiosa $\mathrm{RR}^{\circledR}$ ，CD $219 \mathrm{RR}^{\circledR}$, M-SOY $7908 \mathrm{RR}^{\circledR}$, P98Y11 ${ }^{\circledR}$ e P98R31 ${ }^{\circledR}$ ) e o segundo por herbicidas aplicados de forma isolada ou em mistura: 2,4-D (1340 g ha $\left.\mathrm{ha}^{-1}\right), \quad 2,4-\mathrm{D}$ + glyphosate $\left(1340+1200 \mathrm{~g} \mathrm{ha}^{-1}\right)$, metsulfuronmethyl (3 $\left.\mathrm{g} \mathrm{ha}^{-1}\right)$, metsulfuron-metyl + glyphosate $\left(3+1200 \mathrm{~g} \mathrm{ha}^{-1}\right)$, [MSMA + diuron] $\left(1800+700 \mathrm{~g} \mathrm{ha}^{-1}\right)$, [MSMA + diuron] + glyphosate $\left(1800+700+1200 \mathrm{~g} \mathrm{ha}^{-1}\right)$, [paraquat + diuron] $\left(600+300 \mathrm{~g} \mathrm{ha}^{-1}\right)$, [paraquat + diuron]+glyphosate $(600+300+$ $1200 \mathrm{~g} \mathrm{ha}^{-1}$ ) e uma testemunha sem herbicida.

As plantas daninhas presentes na área experimental foram previamente dessecadas, utilizando-se o herbicida glyphosate, na dosagem equivalente a $1500 \mathrm{~g} \mathrm{ha}^{-1}$. As parcelas experimentais foram constituídas de $4,0 \mathrm{~m}$ de comprimento e seis linhas de semeadura espaçadas de $0,5 \mathrm{~m}$, sendo a área útil representada pelas quatro linhas centrais, descontando-se $0,5 \mathrm{~m}$ das extremidades $\left(6 \mathrm{~m}^{2}\right)$.

A semeadura das variedades de soja RR foi efetuada em 26 de outubro de 2007, na profundidade de $2 \mathrm{~cm}$, distribuindo-se $300 \mathrm{~g}$ de sementes por parcela, equivalendo a perda média de $120 \mathrm{~kg} \mathrm{ha}^{-1}$ de grãos na colheita. Quando as plantas se encontravam em média com três trifólios completamente expandidos, foi realizada aplicação dos tratamentos herbicidas, utilizando-se um pulverizador costal pressurizado com $\mathrm{CO}_{2}$, munido com seis pontas tipo leque TT11002, constituindo um volume de aplicação de $150 \mathrm{~L} \mathrm{ha}^{-1}$.

As avaliações foram realizadas aos 7 , 14, 21 e 42 dias após a aplicação (DAA) para verificar o nível de controle das plantas de soja. Para tanto, empregou-se escala percentual notas, variando de 0 (zero) a 100 (cem) \%, em que 0\% representava a ausência de sintomas e $100 \%$ a morte de todas as plantas. Determinou-se a altura média de dez plantas de soja aos 42 DAA, tomando-se como referência a distância entre o colo das plantas na superfície do solo até a inserção do último trifólio completamente expandido. Nessa data, também foi quantificada a massa seca da parte aérea de dez plantas escolhidas ao acaso na área útil da parcela. Esse material colhido foi acondicionado em estufa de circulação forçada de ar por 72 horas a temperatura média de $65^{\circ} \mathrm{C}$, sendo pesado imediatamente após sua retirada.

Os dados foram submetidos à análise de variância utilizando-se do programa Genes (Cruz, 2006), e as médias comparadas pelo teste de Tukey a $5 \%$ de probabilidade.

\section{Resultados e discussão}


Os tratamentos herbicidas promoveram $\mathrm{D}+$ glyphosate para as variedades $98 \mathrm{Y} 11^{\circledR}$, controle diferenciado das plantas voluntárias P98R31 ${ }^{\circledR}$ e BRS Favorita RR ${ }^{\circledR}$. Estes de soja RR. Aos 7 DAA, observou-se que os resultados corroboram como Bond \& Walker tratamentos contendo [paraquat + diuron] (2009) e Dan et al. (2009), os quais isolados ou em mistura com glyphosate constataram controle médio de $90 \%$ de plantas proporcionaram os maiores níveis de controle voluntárias de soja RR com aplicação de das variedades de soja RR (>93\%) (Tabela 1), paraquat. Os menores níveis de controle nessa não diferindo do tratamento 2,4-D no controle época de avaliação foram verificados com uso da P98Y11 ${ }^{\circledR}$ e P98R $31^{\circledR}$, e do tratamento 2,4- isolado de metsulfuron-methyl.

Tabela 1. Controle (\%) de plantas voluntárias de soja RR aos 7 e 14 dias após a aplicação (DAA) de diversos tratamentos herbicidas. Rio Verde-GO, 2007/2008 ${ }^{(1)}$.

\begin{tabular}{|c|c|c|c|c|c|c|}
\hline \multirow{3}{*}{ Herbicidas } & \multicolumn{6}{|c|}{ Variedades de soja RR } \\
\hline & $\begin{array}{c}\text { M-SOY } \\
\text { 7908RR } \\
\end{array}$ & P98Y11 ${ }^{\circledR}$ & P98R31 ${ }^{\circledR}$ & $\begin{array}{c}\text { BRS Favorita } \\
\mathrm{RR}^{\circledR} \\
\end{array}$ & $\begin{array}{c}\text { BRS Valiosa } \\
\text { RR }^{\circledR} \\
\end{array}$ & CD 219RR ${ }^{\circledR}$ \\
\hline & \multicolumn{6}{|c|}{--- 7 DAA --- } \\
\hline metsulfuron-methyl & 8,05 BCDe & $14,15 \mathrm{BCd}$ & $18,07 \mathrm{Be}$ & $28,70 \mathrm{Ad}$ & $3,87 \mathrm{De}$ & 6,70CDcd \\
\hline $\begin{array}{l}\text { metsulfuron-methyl + } \\
\text { glyphosate }\end{array}$ & 7,20 De & $33,70 \mathrm{Bc}$ & 44,12 Ad & $22,47 \mathrm{Cd}$ & $10,30 \mathrm{De}$ & $14,52 \mathrm{CDc}$ \\
\hline $2,4-\mathrm{D}$ & $31,65 \mathrm{Dd}$ & $96,02 \mathrm{Aa}$ & $96,80 \mathrm{Aa}$ & $87,80 \mathrm{ABb}$ & $58,72 \mathrm{Cd}$ & $81,30 \mathrm{Bb}$ \\
\hline 2,4-D + glyphosate & $74,80 \mathrm{BCc}$ & $99,15 \mathrm{Aa}$ & $95,52 \mathrm{Aa}$ & 99,47 Аa & $68,77 \mathrm{Ccd}$ & $80,45 \mathrm{Bb}$ \\
\hline [MSMA+diuron] & $75,40 \mathrm{ABc}$ & $69,97 \mathrm{Bb}$ & $54,97 \mathrm{Cc}$ & $75,62 \mathrm{ABc}$ & 76,70ABbc & $81,45 \mathrm{Ab}$ \\
\hline $\begin{array}{l}\text { [MSMA+diuron] + } \\
\text { glyphosate }\end{array}$ & $86,98 \mathrm{ABb}$ & $77,12 \mathrm{BCb}$ & $76,05 \mathrm{Bb}$ & $88,12 \mathrm{Ab}$ & $80,97 \mathrm{ABCb}$ & $87,85 \mathrm{Ab}$ \\
\hline [paraquat+diuron] & 93,10 Aab & $95,70 \mathrm{Aa}$ & $100,00 \mathrm{Aa}$ & $100,00 \mathrm{Aa}$ & $100,00 \mathrm{Aa}$ & $100,00 \mathrm{Aa}$ \\
\hline $\begin{array}{l}\text { [paraquat+diuron] + } \\
\text { glyphosate }\end{array}$ & $100,00 \mathrm{Aa}$ & $98,02 \mathrm{Aa}$ & $98,37 \mathrm{Aa}$ & $100,00 \mathrm{Aa}$ & $100,00 \mathrm{Aa}$ & $100,00 \mathrm{Aa}$ \\
\hline \multirow[t]{2}{*}{ testemunha } & $0,00 \mathrm{Ae}$ & $0,00 \mathrm{Ae}$ & $0,00 \mathrm{Af}$ & $0,00 \mathrm{Ae}$ & $0,00 \mathrm{Ae}$ & $0,00 \mathrm{Ad}$ \\
\hline & \multicolumn{6}{|c|}{--- 14 DAA --- } \\
\hline metsulfuron-methyl & $13,55 \mathrm{De}$ & $17,75 \mathrm{CDc}$ & $33,25 \mathrm{Ad}$ & $29,00 \mathrm{ABd}$ & $21,50 \mathrm{BCe}$ & $27,00 \mathrm{ABd}$ \\
\hline $\begin{array}{l}\text { metsulfuron-methyl + } \\
\text { glyphosate }\end{array}$ & $34,97 \mathrm{Cd}$ & $79,00 \mathrm{Ab}$ & $78,75 \mathrm{Ab}$ & $51,25 \mathrm{Bc}$ & $41,75 \mathrm{Cd}$ & $40,25 \mathrm{Cc}$ \\
\hline 2,4-D & $94,55 \mathrm{ABab}$ & $99,25 \mathrm{Aa}$ & $100,00 \mathrm{Aa}$ & $92,00 \mathrm{BCa}$ & $85,00 \mathrm{Cc}$ & $98,75 \mathrm{ABa}$ \\
\hline 2,4-D + glyphosate & $99,75 \mathrm{Aa}$ & $100,00 \mathrm{Aa}$ & $100,00 \mathrm{Aa}$ & $100,00 \mathrm{Aa}$ & $94,25 \mathrm{Aab}$ & $100,00 \mathrm{Aa}$ \\
\hline [MSMA + diuron] & $60,50 \mathrm{Cc}$ & $72,00 \mathrm{Bb}$ & $50,00 \mathrm{Dc}$ & $77,00 \mathrm{Bb}$ & $78,75 \mathrm{ABc}$ & $86,25 \mathrm{Ab}$ \\
\hline $\begin{array}{l}{[\text { MSMA + diuron }]+} \\
\text { glyphosate }\end{array}$ & $87,80 \mathrm{Bb}$ & $73,25 \mathrm{Cb}$ & $77,75 \mathrm{Cb}$ & $92,00 \mathrm{Aba}$ & $86,25 \mathrm{Bbc}$ & $97,75 \mathrm{Aa}$ \\
\hline [paraquat + diuron] & $100,00 \mathrm{Aa}$ & $98,50 \mathrm{Aa}$ & $100,00 \mathrm{Aa}$ & $100,00 \mathrm{Aa}$ & $100,00 \mathrm{Aa}$ & $100,00 \mathrm{Aa}$ \\
\hline $\begin{array}{l}\text { [paraquat + diuron] + } \\
\text { glyphosate }\end{array}$ & $100,00 \mathrm{Aa}$ & $98,25 \mathrm{Aa}$ & $99,65 \mathrm{Aa}$ & $100,00 \mathrm{Aa}$ & $100,00 \mathrm{Aa}$ & $100,00 \mathrm{Aa}$ \\
\hline testemunha & 0,00 Af & $0,00 \mathrm{Ad}$ & $0,00 \mathrm{Ae}$ & $0,00 \mathrm{Ae}$ & $0,00 \mathrm{Af}$ & $0,00 \mathrm{Ae}$ \\
\hline
\end{tabular}

(1)Médias seguidas pela mesma letra minúscula na coluna e maiúscula na linha não diferem entre si pelo teste de Tukey a 5\% de probabilidade. 
A adição de glyphosate ao metsulfuronmethyl promoveu acréscimos no controle das variedades P98Y $11^{\circledR}$ e P98R $31^{\circledR}$ aos 7 DAA, o que também ocorreu com 2,4-D sobre M-SOY 7908 RR $^{\circledR}$ e BRS Favorita RR ${ }^{\circledR}$ e com [MSMA + diuron] sobre M-SOY $7908 \mathrm{RR}^{\circledR}$, P98R31 ${ }^{\circledR}$, BRS Favorita RR ${ }^{\circledR}$ e BRS Valiosa RR ${ }^{\circledR}$ (Tabela 1). É importante ressaltar que quanto mais rápido é o controle das plantas voluntárias de soja, mais cedo é interrompida a multiplicação do fungo causador da ferrugem asiática, destacando a importância do herbicida não só em ocasionar a morte das plantas tigueras como também de antecipar os efeitos da dessecação. Este resultado obtido com paraquat está relacionado ao seu mecanismo de ação, que captura os elétrons provenientes da respiração, gerando radicais tóxicos instáveis que sofrem rapidamente auto-oxidação (Santos et al., 2006a), promovendo a degradação de membranas pela peroxidação de lipídios, que ocasionará a morte celular.

A eficácia dos tratamentos herbicidas no controle das plantas voluntárias de soja RR foi variável, conforme o genótipo. Na avaliação de 7 DAA, constatou-se que todas as variedades foram altamente controladas por [paraquat + diuron], independentemente da mistura com glyphosate (Tabela 1). As variedades P98Y11 ${ }^{\circledR}$, P98R $31^{\circledR}$ e BRS Favorita $\mathrm{RR}^{\circledR}$ foram as mais sensíveis aos tratamentos contendo 2,4-D, no entanto P98Y11 ${ }^{\circledR} \mathrm{e}$ P98R31 ${ }^{\circledR}$ apresentaram o maior nível de tolerância aos tratamentos contendo a mistura pré-formulada [MSMA + diuron].

Decorridas duas semanas após a aplicação dos herbicidas, pode-se constatar que a adição do glyphosate aos outros herbicidas não acarretou prejuízos ao controle das plantas voluntárias de soja RR (Tabela 1). A adição de glyphosate ao [MSMA + diuron] gerou ganhos na eficácia de controle das variedades CD 219 RR $^{\circledR}$, BRS Favorita RR ${ }^{\circledR}$, M-SOY 7908 RR $^{\circledR} \mathrm{e}$ P98R3 $11^{\circledR}$. O mesmo efeito foi observado com a variedade BRS Valiosa RR $^{\circledR}$ quando submetida ao 2,4-D e para as demais variedades quando associado ao metsulfuron-methyl.

A possível justificativa para o efeito sinérgico do glyphosate no controle de plantas de soja RR pode estar relacionada a alta capacidade da formulação desse herbicida em penetrar a cutícula das membranas celulares presente nas células da epiderme foliar. Segundo Santos et al. (2007), são disponibilizadas no mercado diversas formulações de glyphosate, que, apesar de apresentarem o mesmo mecanismo de ação, possuem, na composição, diferentes sais, sendo os principais: sal potássico, de isopropilamina e de amônio. Stock \& Holloway (1993) afirmaram que surfactantes, além de melhorarem a deposição e retenção do herbicida nas plantas, podem penetrar na cutícula, às vezes rapidamente, ocasionando aumento da permeabilidade da mesma em baixas concentrações ou danificando-a quando a deposição ocorre em maiores concentrações. Alterações apenas na formulação de um mesmo princípio ativo podem causar maior intoxicação a organismos não-alvo, principalmente em relação à microbiota do solo (Santos et al., 2005; 2006b), maior velocidade na translocação (Molin \& Hirase, 2005) e melhor controle de algumas espécies de plantas daninhas (Jakelaits et al., 2001; Molin \& Hirase, 2004; Li et al., 2005).

Aos 14 DAA, os melhores tratamentos no controle das plantas voluntárias de soja RR foram [paraquat + diuron], [paraquat + diuron] + glyphosate, 2,4-D + glyphosate e 2,4-D. Esse último apenas não apresentou a mesma eficácia no controle da variedade BRS Valiosa $R^{\circledR}$ (Tabela 1). Já [MSMA + diuron] + glyphosate apresentou controle acima de $90 \%$ para as variedades CD 219 RR $^{\circledR}$ e BRS Favorita RR ${ }^{\circledR}$. É relevante salientar a evolução no controle das plantas voluntárias de soja RR visualizada no tratamento metsulfuron-methyl + glyphosate, chegando a atingir níveis médios de controle de até $79 \%$, sendo que as variedades P98Y $11^{\circledR} \mathrm{e}$ 
P98R $311^{\circledR}$ mostraram maior sensibilidade nessa avaliação.

Aos 21 DAA, registrou-se a morte de todas as plantas de soja voluntária nas parcelas que receberam o tratamento 2,4-D + glyphosate (Tabela 2). Os tratamentos 2,4-D, [paraquat + diuron] e [paraquat + diuron] + glyphosate também continuaram se destacando no controle dessas plantas, inclusive não diferindo estatisticamente do tratamento 2,4-D + glyphosate.

Tabela 2. Controle (\%) de plantas voluntárias de soja RR aos 21 e 42 dias após a aplicação (DAA) de diversos tratamentos herbicidas. Rio Verde-GO, 2007/2008 ${ }^{(1)}$.

\begin{tabular}{|c|c|c|c|c|c|c|}
\hline \multirow{3}{*}{ Herbicidas } & \multicolumn{6}{|c|}{ Variedades de soja RR } \\
\hline & $\begin{array}{c}\text { M-SOY } \\
\text { 7908RR }{ }^{\circledR} \\
\end{array}$ & P98Y11 ${ }^{\circledR}$ & $\mathrm{P}^{2} 8 \mathrm{R} 31^{\circledR}$ & $\begin{array}{c}\text { BRS Favorita } \\
\text { RR }^{\circledR} \\
\end{array}$ & $\begin{array}{c}\text { BRS Valiosa } \\
\text { RR }^{\circledR} \\
\end{array}$ & CD 219RR ${ }^{\circledR}$ \\
\hline & \multicolumn{6}{|c|}{--- 21 DAA --- } \\
\hline metsulfuron-methyl & $15,35 \mathrm{Cd}$ & $17,00 \mathrm{BCd}$ & $26,30 \mathrm{ABc}$ & $25,55 \mathrm{ABd}$ & $26,07 \mathrm{ABd}$ & $31,55 \mathrm{Ad}$ \\
\hline $\begin{array}{l}\text { metsulfuron-methyl + } \\
\text { glyphosate }\end{array}$ & $58,63 \mathrm{Bc}$ & $79,13 \mathrm{Ab}$ & $86,63 \mathrm{Ab}$ & $59,55 \mathrm{Bc}$ & $52,70 \mathrm{Bc}$ & $57,88 \mathrm{Bc}$ \\
\hline $2,4-\mathrm{D}$ & 99,00 Aab & $100,00 \mathrm{Aa}$ & 100,00 Aa & $97,63 \mathrm{Aa}$ & $91,38 \mathrm{Aa}$ & 99,83 Aa \\
\hline 2,4-D + glyphosate & $100,00 \mathrm{Aa}$ & $100,00 \mathrm{Aa}$ & 100,00 Aa & $100,00 \mathrm{Aa}$ & $100,00 \mathrm{Aa}$ & $100,00 \mathrm{Aa}$ \\
\hline [MSMA+diuron] & $55,83 \mathrm{Bc}$ & $41,23 \mathrm{Cc}$ & $19,28 \mathrm{Dc}$ & $79,98 \mathrm{Ab}$ & $16,40 \mathrm{Dd}$ & $89,18 \mathrm{Ab}$ \\
\hline $\begin{array}{l}\text { [MSMA+diuron] + } \\
\text { glyphosate }\end{array}$ & $89,65 \mathrm{Ab}$ & $70,40 \mathrm{Bb}$ & $79,18 \mathrm{Bb}$ & $91,05 \mathrm{Aab}$ & $74,50 \mathrm{Bc}$ & $98,65 \mathrm{Aab}$ \\
\hline [paraquat+diuron] & $100,00 \mathrm{Aa}$ & $97,73 \mathrm{Aa}$ & $100,00 \mathrm{Aa}$ & $100,00 \mathrm{Aa}$ & $100,00 \mathrm{Aa}$ & $100,00 \mathrm{Aa}$ \\
\hline $\begin{array}{l}\text { [paraquat+diuron] + } \\
\text { glyphosate }\end{array}$ & $100,00 \mathrm{Aa}$ & $98,50 \mathrm{Aa}$ & $99,40 \mathrm{Aa}$ & $100,00 \mathrm{Aa}$ & $100,00 \mathrm{Aa}$ & $100,00 \mathrm{Aa}$ \\
\hline \multirow[t]{2}{*}{ testemunha } & $0,00 \mathrm{Ae}$ & $0,00 \mathrm{Ae}$ & $0,00 \mathrm{Ad}$ & $0,00 \mathrm{Ae}$ & $0,00 \mathrm{Ae}$ & $0,00 \mathrm{Ae}$ \\
\hline & \multicolumn{6}{|c|}{-- 42 DAA --- } \\
\hline metsulfuron-methyl & $21,02 \mathrm{ABc}$ & $17,90 \mathrm{Bd}$ & $26,22 \mathrm{ABc}$ & $24,52 \mathrm{ABc}$ & $31,22 \mathrm{Ad}$ & $19,95 \mathrm{ABb}$ \\
\hline $\begin{array}{l}\text { metsulfuron-methyl + } \\
\text { glyphosate }\end{array}$ & $92,12 \mathrm{ABa}$ & $98,47 \mathrm{Aa}$ & 97,72 Аa & $93,30 \mathrm{Aa}$ & $81,20 \mathrm{Bb}$ & $90,62 \mathrm{ABa}$ \\
\hline 2,4-D & $100,00 \mathrm{Aa}$ & $100,00 \mathrm{Aa}$ & $100,00 \mathrm{Aa}$ & $100,00 \mathrm{Aa}$ & 91,30 Aab & $100,00 \mathrm{Aa}$ \\
\hline 2,4-D + glyphosate & $100,00 \mathrm{Aa}$ & $100,00 \mathrm{Aa}$ & $100,00 \mathrm{Aa}$ & $100,00 \mathrm{Aa}$ & $100,00 \mathrm{Aa}$ & $100,00 \mathrm{Aa}$ \\
\hline [MSMA+diuron] & $65,80 \mathrm{Bb}$ & $31,22 \mathrm{Cc}$ & $23,82 \mathrm{CDc}$ & $30,77 \mathrm{Cc}$ & $16,00 \mathrm{De}$ & $97,62 \mathrm{Aa}$ \\
\hline $\begin{array}{l}{[\text { MSMA+diuron }]+} \\
\text { glyphosate }\end{array}$ & $73,55 \mathrm{Bb}$ & $67,27 \mathrm{Bb}$ & $42,77 \mathrm{Cb}$ & $67,20 \mathrm{Bb}$ & $53,87 \mathrm{Cc}$ & $100,00 \mathrm{Aa}$ \\
\hline [paraquat+diuron] & $100,00 \mathrm{Aa}$ & $100,0 \mathrm{Aa}$ & $100,00 \mathrm{Aa}$ & $100,00 \mathrm{Aa}$ & $100,00 \mathrm{Aa}$ & $100,00 \mathrm{Aa}$ \\
\hline $\begin{array}{l}\text { [paraquat+diuron] + } \\
\text { glyphosate }\end{array}$ & $100,00 \mathrm{Aa}$ & $100,00 \mathrm{Aa}$ & $100,00 \mathrm{Aa}$ & $100,00 \mathrm{Aa}$ & $100,00 \mathrm{Aa}$ & $100,00 \mathrm{Aa}$ \\
\hline testemunha & $0,00 \mathrm{Ad}$ & $0,00 \mathrm{Ae}$ & $0,00 \mathrm{Ad}$ & $0,00 \mathrm{Ad}$ & $0,00 \mathrm{Af}$ & $0,00 \mathrm{Ac}$ \\
\hline
\end{tabular}

${ }^{(1)}$ Médias seguidas pela mesma letra minúscula na coluna e maiúscula na linha não diferem entre si pelo teste de Tukey a 5\% de probabilidade.

$\mathrm{O}$ [paraquat + diuron] apenas não diuron] + glyphosate essa situação não ocorreu promoveu controle total da variedade apenas nas variedades P98Y11 ${ }^{\circledR}$ e P98R31 ${ }^{\circledR}$. P98Y11 ${ }^{\circledR}$, enquanto que para [paraquat + Contudo, os sintomas de dano observados 
nessas variedades foram de alta intensidade, impossibilitando qualquer tipo de recuperação das plantas. Estes resultados corroboram como os obtidos por Bond \& Walker (2009) ao verificarem que a aplicação de paraquat proporcionou controle médio de $95 \%$ das plantas voluntárias de soja RR.

Os efeitos verificados nos tratamentos contendo [MSMA + diuron], seja isolado ou em mistura ao glyphosate, oscilaram entre as variedades, porém a presença do glyphosate foi positiva para o controle de quatro das seis variedades avaliadas (M-SOY 7908 RR $^{\circledR}$, P98Y11 ${ }^{\circledR}$, P98R31 ${ }^{\circledR}$ e BRS Valiosa RR ${ }^{\circledR}$ ). Petter et al. (2007) observaram que a adição do glyphosate junto a calda de aplicação do inseticida spinosad aumentou a atividade residual desse inseticida no controle da lagartada-soja. Esse efeito foi atribuído pelos autores a maior absorção foliar do spinosad, promovida pela ação dos ingredientes da formulação do glyphosate, acelerando a retirada do inseticida da superfície foliar, onde suas moléculas ficariam mais suscetíveis à lavagem pelas gotas da chuva. Controle variando de $19 \%$ (P98R31 ${ }^{\circledR}$ ) até $89 \%\left(\mathrm{CD} 219 \mathrm{RR}^{\circledR}\right.$ ) foram observados quando se aplicou a mistura préformulada [MSMA + diuron].

O glyphosate potencializou a ação do metsulfuron-methyl em todas as variedades de soja RR aos 21 DAA (Tabela 2). Nessa mistura foi verificada ação mais pronunciada do glyphosate no favorecimento da atuação do metsulfuron-methyl. A resposta observada na variedade P98R31 ${ }^{\circledR}$ elucida essa constatação. O nível de controle da aplicação do metsulfuronmethyl isolado sobre essa variedade foi de $26 \%$, passando para mais de $86 \%$, quando se adicionou o glyphosate na calda de aplicação. $O$ controle quando se utilizou somente o metsulfuron-methyl não chegou a $32 \%$, para qualquer uma das variedades avaliadas.

Aos 42 DAA, constatou-se que os tratamentos herbicidas 2,4-D + glyphosate, [paraquat + diuron] e [paraquat + diuron] + glyphosate proporcionaram a morte de $100 \%$ das plantas, independentemente da variedade avaliada (Tabela 2). Esses resultados permitem afirmar que, na mistura em tanque, o glyphosate não prejudica a ação do paraquat. $\mathrm{O}$ que ocorre em outras situações é que devido a rapidez de ação o paraquat reduz a eficiência do glyphosate, prejudicando a translocação do glyphosate para os meristemas, dificultando assim sua atividade. A aplicação apenas de 2,4D não apresentou controle total somente da BRS Valiosa $\mathrm{RR}^{\circledR}$, contudo foi superior a $91 \%$. O uso de [MSMA + diuron], seja isolado ou em mistura com glyphosate foi eficiente apenas no controle das tigueras da CD $219 \mathrm{RR}^{\circledR}$.

A mistura de metsulfuron-methyl com glyphosate apresentou controle acima de $90 \%$ para todas as variedades RR aos 42 DAA, exceto para a BRS Valiosa RR $^{\circledR}$ (Tabela 2). Esse tratamento herbicida demonstrou ter ação mais lenta, em comparação aos que continham paraquat ou 2,4-D. Por outro lado, a aplicação isolada de metsulfuron-methyl não foi eficaz no controle das variedades $R^{\circledR}{ }^{\circledR}$. É marcante o fato de que a adição do glyphosate promoveu maior controle de uma espécie vegetal que possui um gene de tolerância a esse herbicida. A resposta deve estar nos ingredientes da formulação de glyphosate (Roundup Transorb $^{\circledR}$ ), podendo favorecer a penetração das moléculas do metsulfuron-methyl nas folhas da soja RR. Em geral, a CD 219 RR $^{\circledR}$ foi a que apresentou suscetibilidade ao maior número de tratamentos herbicidas. Santos et al. (2007), quantificando a intoxicação de plantas da CD 219 RR $^{\circledR}$ após a aplicação de formulações de glyphosate, verificaram leve efeito tóxico do Roundup Ready ${ }^{\circledR}(25 \%)$, seguido por Zapp Qi ${ }^{\circledR}(28 \%)$ e maiores danos causados por Roundup Transorb ${ }^{\circledR}(40 \%)$. Os mesmos autores observaram que o Roundup Transorb $^{\circledR}$ reduziu o número de nódulos radiculares das plantas. 
Quando se analisou o efeito dos provocaram redução dessa característica em herbicidas sobre a altura de plantas, observou- todas variedades (Tabela 3). se que todos os tratamentos herbicidas

Tabela 3. Altura de plantas $(\mathrm{cm})$ e massa seca da parte aérea $\left(\mathrm{g} \mathrm{planta}^{-1}\right)$ de plantas voluntárias de soja RR aos 42 DAA de diversos tratamentos herbicidas. Rio Verde-GO, 2007/2008 ${ }^{(1)}$.

\begin{tabular}{|c|c|c|c|c|c|c|}
\hline \multirow{3}{*}{ Herbicidas } & \multicolumn{6}{|c|}{ Variedades de soja RR } \\
\hline & $\begin{array}{c}\text { M-SOY } \\
\text { 7908RR } \\
\end{array}$ & P98Y11 ${ }^{\circledR}$ & P98R31 ${ }^{\circledR}$ & $\begin{array}{c}\text { BRS Favorita } \\
\mathrm{RR}^{\circledR}\end{array}$ & $\begin{array}{c}\text { BRS Valiosa } \\
\text { RR }^{\circledR} \\
\end{array}$ & $\mathrm{CD}_{219 \mathrm{RR}^{\circledR}}$ \\
\hline & \multicolumn{6}{|c|}{--- Altura de plantas --- } \\
\hline metsulfuron-methyl & $30,75 \mathrm{Bb}$ & $48,10 \mathrm{Ab}$ & $13,25 \mathrm{Bc}$ & $43,57 \mathrm{Ab}$ & $29,22 \mathrm{Bb}$ & $33,90 \mathrm{Bb}$ \\
\hline $\begin{array}{l}\text { metsulfuron- } \\
\text { methyl+glyphosate }\end{array}$ & $16,82 \mathrm{ABc}$ & $14,25 \mathrm{Bc}$ & $22,47 \mathrm{Ad}$ & $14,15 \mathrm{Bcd}$ & $15,82 \mathrm{ABc}$ & $16,40 \mathrm{ABc}$ \\
\hline 2,4-D & $10,30 \mathrm{ABc}$ & $7,85 \mathrm{Bc}$ & $15,45 \mathrm{Ad}$ & $12,32 \mathrm{ABcd}$ & $9,32 \mathrm{ABc}$ & $10,42 \mathrm{ABc}$ \\
\hline 2,4-D+glyphosate & $12,27 \mathrm{ABc}$ & $10,37 \mathrm{ABc}$ & $17,15 \mathrm{Ad}$ & $12,10 \mathrm{ABcd}$ & $9,27 \mathrm{Bc}$ & $14,52 \mathrm{ABc}$ \\
\hline [MSMA+diuron] & $26,87 \mathrm{Cb}$ & $42,75 \mathrm{Bb}$ & $50,52 \mathrm{Ab}$ & $12,92 \mathrm{Dcd}$ & $25,87 \mathrm{Cb}$ & $12,97 \mathrm{Dc}$ \\
\hline $\begin{array}{l}\text { [MSMA+diuron]+ } \\
\text { glyphosate }\end{array}$ & $15,27 \mathrm{Bc}$ & $14,67 \mathrm{Bc}$ & $49,17 \mathrm{Ab}$ & $15,60 \mathrm{Bc}$ & $14,27 \mathrm{Bc}$ & $11,82 \mathrm{Bc}$ \\
\hline [paraquat+diuron] & $14,60 \mathrm{Ac}$ & $11,00 \mathrm{Ac}$ & $16,05 \mathrm{Ad}$ & 9,10 Acd & $13,67 \mathrm{Ac}$ & $11,05 \mathrm{Ac}$ \\
\hline $\begin{array}{l}\text { [paraquat+diuron]+ } \\
\text { glyphosate }\end{array}$ & $15,25 \mathrm{Ac}$ & $13,07 \mathrm{ABc}$ & $17,65 \mathrm{Ad}$ & $6,87 \mathrm{Bd}$ & $14,25 \mathrm{ABc}$ & $16,10 \mathrm{Ac}$ \\
\hline \multirow[t]{2}{*}{ testemunha } & $78,92 \mathrm{Ba}$ & $91,84 \mathrm{Aa}$ & $92,55 \mathrm{Aa}$ & $85,90 \mathrm{Aba}$ & $78,67 \mathrm{Ba}$ & $82,70 \mathrm{Ba}$ \\
\hline & \multicolumn{6}{|c|}{--- Massa seca da parte aérea --- } \\
\hline metsulfuron-methyl & $43,37 \mathrm{BCb}$ & $132,00 \mathrm{Ab}$ & $69,60 \mathrm{Bb}$ & $34,72 \mathrm{Cab}$ & $50,37 \mathrm{BCbc}$ & $51,17 \mathrm{BCb}$ \\
\hline $\begin{array}{l}\text { metsulfuron- } \\
\text { methyl+glyphosate }\end{array}$ & $16,65 \mathrm{Abc}$ & 25,67 Ade & $19,35 \mathrm{Ac}$ & $28,62 \mathrm{Aab}$ & 22,22 Acd & $24,07 \mathrm{Abc}$ \\
\hline 2,4-D & $14,17 \mathrm{Abc}$ & $13,25 \mathrm{Ae}$ & $13,25 \mathrm{Ac}$ & $16,90 \mathrm{Ab}$ & $12,80 \mathrm{Ad}$ & $14,97 \mathrm{Ac}$ \\
\hline 2,4-D+glyphosate & $12,72 \mathrm{Abc}$ & $15,27 \mathrm{Ae}$ & $14,60 \mathrm{Ac}$ & $16,55 \mathrm{Ab}$ & $14,32 \mathrm{Ad}$ & $16,32 \mathrm{Ac}$ \\
\hline [MSMA+diuron] & $25,40 \mathrm{Bbc}$ & $74,40 \mathrm{Ac}$ & $83,60 \mathrm{Aab}$ & $27,10 \mathrm{Bab}$ & $76,20 \mathrm{Ab}$ & $17,10 \mathrm{Bc}$ \\
\hline $\begin{array}{l}\text { [MSMA+diuron]+ } \\
\text { glyphosate }\end{array}$ & $11,52 \mathrm{Bc}$ & 54,57 Acd & $21,60 \mathrm{Bc}$ & $28,90 \mathrm{ABab}$ & $23,60 \mathrm{Bcd}$ & $13,05 \mathrm{Bc}$ \\
\hline [paraquat+diuron] & $10,62 \mathrm{Ac}$ & $12,77 \mathrm{Ae}$ & $12,27 \mathrm{Ac}$ & $11,35 \mathrm{Ab}$ & $11,10 \mathrm{Ad}$ & $14,37 \mathrm{Ac}$ \\
\hline $\begin{array}{l}\text { [paraquat+diuron]+ } \\
\text { glyphosate }\end{array}$ & $10,82 \mathrm{Ac}$ & $11,22 \mathrm{Ae}$ & $12,57 \mathrm{Ac}$ & $13,15 \mathrm{Ab}$ & $11,20 \mathrm{Ad}$ & $12,50 \mathrm{Ac}$ \\
\hline testemunha & $125,40 \mathrm{BCa}$ & $375,57 \mathrm{Aa}$ & $107,02 \mathrm{Ca}$ & $49,87 \mathrm{Da}$ & $140,42 \mathrm{Ba}$ & $348,70 \mathrm{Aa}$ \\
\hline
\end{tabular}

${ }^{(1)}$ Médias seguidas pela mesma letra, minúscula na coluna e maiúscula na linha, não diferem entre si pelo teste de Tukey a 5\% de probabilidade.

A adição do glyphosate ao metsulfuron- dessa mistura no controle da soja RR. No methyl promoveu maior inibição no porte da entanto, a presença do glyphosate junto ao soja quando comparado a aplicação isolada [MSMA + diuron] ocasionou reduções desse herbicida inibidor da enzima acetolactato significativas na altura de plantas apenas das sintase (ALS), comprovando a importância variedades M-SOY 7908 RR ${ }^{\circledR}$, P98Y11 e BRS 
Valiosa $\mathrm{RR}^{\circledR}$. Os tratamentos 2,4-D e [paraquat + diuron] foram muito eficazes em reduzir a alturas das plantas de soja RR, não respondendo a adição do glyphosate. É importante ressaltar que em situações de semeadura de milho safrinha após o cultivo de soja RR, onde se faz necessário o controle das tigueras de soja em curto período de tempo, visando não atrasar a semeadura de segunda safra, a opção do 2,4-D deve ser visto com cautela. A aplicação desse herbicida pode deixar resíduos no solo, causando intoxicação nas plântulas da cultura de sucessão.

O menor efeito na inibição do crescimento na altura das plantas das variedades P98Y11 ${ }^{\circledR}$, BRS Favorita RR ${ }^{\circledR}, \mathrm{CD}$ $219 \mathrm{RR}^{\circledR}$, M-SOY $7908 \mathrm{RR}^{\circledR}$ e BRS Valiosa $\mathrm{RR}^{\circledR}$ foi observado para metsulfuron-methyl (Tabela 3). Para as duas últimas variedades, este tratamento não diferiu do [MSMA + diuron]. O valor obtido com P98R $31^{\circledR}$ apresentou menor redução com os tratamentos [MSMA + diuron] e [MSMA + diuron] + glyphosate.

Para o acúmulo de matéria seca, os tratamentos [paraquat + diuron], [paraquat + diuron] + glyphosate, 2,4-D e 2,4-D + glyphosate promoveram reduções significativas em todas as variedades de soja RR (Tabela 3 ). A adição de glyphosate ao [MSMA + diuron] acarretou em maior redução no acúmulo de matéria seca para P98R31 ${ }^{\circledR}$ e BRS Valiosa $\mathrm{RR}^{\circledR}$, e apenas não diferiu da testemunha para BRS Favorita $R^{\circledR}{ }^{\circledR}$. O glyphosate quando aplicado em mistura com metsulfuron-methyl também promoveu menor acúmulo de fitomassa das variedades P98Y $11^{\circledR}$ e P98R $31^{\circledR}$. Stock \& Holloway (1993) afirmaram que os surfactantes melhoram a deposição e a retenção do herbicida nas plantas, e que a penetração na cutícula ocasiona o aumento da permeabilidade em baixas concentrações, ou danificando-a quando a deposição ocorre em maiores concentrações.
Assim, fica evidente que a sensibilidade da soja RR é diferenciada em função do tratamento herbicida. Dessa forma, recomendase para cada cultivar a realização de ensaios visando determinar o herbicida mais adequado para dessecação. Constatou-se também que o glyphosate não prejudicou a ação de nenhum dos tratamentos herbicidas avaliados, pelo contrário, em determinados casos promoveu ganhos no controle das plantas de soja. Com isso, é possível associar em uma única operação o controle das tigueras de soja RR com o manejo das plantas daninhas em póscolheita. Contudo, trabalhos devem ser realizados para verificar se os herbicidas utilizados para dessecação da soja RR não prejudicam a eficiência do glyphosate nas plantas daninhas após a colheita, fato que pode ocorrer, principalmente se a associação for feita em tratamento contendo o herbicida paraquat. Caso o objetivo for apenas o controle da soja RR tiguera é possível dispensar a adição do glyphosate, quando se optar pelos tratamentos [paraquat + diuron] ou 2,4-D.

Destaca-se que foram avaliados cinco mecanismos de ação de herbicidas para controle das plantas voluntárias de soja RR: inibidores de ALS (metsulfuron-methyl); mimetizadores de auxinas (2,4-D); mecanismo de ação desconhecido (MSMA); inibidores de fotossíntese, atuando no Fotossistema I (paraquat); e Fotossistema II (diuron), sendo esses mecanismos de ação distintos do glyphosate (Inibidor da EPSPs). Desta forma, os tratamentos herbicidas apresentaram eficácias diferenciadas para o controle das variedades RR (Tabela 4). Estas opções possibilitam a alternância de mecanismos de ação, reduzindo o risco de seleção de biótipos resistentes de plantas daninhas. 
Tabela 4. Tratamentos herbicidas para o controle de plantas voluntárias de soja RR, provenientes de diferentes variedades. Rio Verde-GO, 2007/2008.

\begin{tabular}{|c|c|c|}
\hline $\begin{array}{l}\text { Variedade } \\
\text { de soja RR }\end{array}$ & $\begin{array}{l}\text { Tratamentos herbicidas que } \\
\text { proporcionaram controle superior a } 90 \%\end{array}$ & $\begin{array}{l}\text { Mecanismos de ação com possibilidade } \\
\text { de uso (excluindo o glyphosate) }\end{array}$ \\
\hline $\begin{array}{l}\text { M-SOY } \\
\text { 7908RR }\end{array}$ & $\begin{array}{l}\text { [paraquat+diuron]; } \\
\text { [paraquat+diuron]+glyphosate; } 2,4-\mathrm{D} ; \\
\text { 2,4-D+glyphosate; metsulfuron- } \\
\text { methyl+glyphosate. }\end{array}$ & $\begin{array}{l}\text { Inibidores do Fotossistema I; Inibidores } \\
\text { do Fotossistema II; Mimetizadores de } \\
\text { auxinas; Inibidores da ALS }\end{array}$ \\
\hline P98Y11 $^{\circledR}$ & $\begin{array}{l}\text { [paraquat+diuron]; } \\
\text { [paraquat+diuron]+glyphosate; } 2,4-\mathrm{D} ; \\
\text { 2,4-D+glyphosate; metsulfuron- } \\
\text { methyl+glyphosate. }\end{array}$ & $\begin{array}{l}\text { Inibidores do Fotossistema I; Inibidores } \\
\text { do Fotossistema II; Mimetizadores de } \\
\text { auxinas; Inibidores da ALS }\end{array}$ \\
\hline P98R31 ${ }^{\circledR}$ & $\begin{array}{l}\text { [paraquat+diuron]; } \\
\text { [paraquat+diuron]+glyphosate; } 2,4-\mathrm{D} ; \\
\text { 2,4-D+glyphosate; metsulfuron- } \\
\text { methyl+glyphosate. }\end{array}$ & $\begin{array}{l}\text { Inibidores do Fotossistema I; Inibidores } \\
\text { do Fotossistema II; Mimetizadores de } \\
\text { auxinas; Inibidores da ALS }\end{array}$ \\
\hline $\begin{array}{c}\text { BRS } \\
\text { FavoritaRR }^{\circledR}\end{array}$ & $\begin{array}{l}\text { [paraquat+diuron]; } \\
\text { [paraquat+diuron]+glyphosate; } 2,4-\mathrm{D} ; \\
\text { 2,4-D+glyphosate; metsulfuron- } \\
\text { methyl+glyphosate. }\end{array}$ & $\begin{array}{l}\text { Inibidores do Fotossistema I; Inibidores } \\
\text { do Fotossistema II; Mimetizadores de } \\
\text { auxinas; Inibidores da ALS }\end{array}$ \\
\hline $\begin{array}{c}\text { BRS } \\
\text { ValiosaRR }^{\circledR}\end{array}$ & $\begin{array}{c}\text { [paraquat+diuron]; } \\
\text { [paraquat+diuron]+glyphosate; } 2,4-\mathrm{D} ; \\
\text { 2,4-D+glyphosate. }\end{array}$ & $\begin{array}{c}\text { Inibidores do Fotossistema I; Inibidores } \\
\text { do Fotossistema II; Mimetizadores de } \\
\text { auxinas }\end{array}$ \\
\hline $\mathrm{CD} 19 \mathrm{RR}^{\circledR}$ & $\begin{array}{c}\text { [paraquat+diuron]; } \\
\text { [paraquat+diuron]+glyphosate; 2,4-D; } \\
\text { 2,4-D + glyphosate; metsulfuron- } \\
\text { methyl+glyphosate; [MSMA+diuron]; } \\
\text { [MSMA+diuron]+glyphosate. }\end{array}$ & $\begin{array}{c}\text { Inibidores do Fotossistema I; Inibidores } \\
\text { do Fotossistema II; Mimetizadores de } \\
\text { auxinas; Inibidores da ALS; Mecanismo } \\
\text { desconhecido (MSMA) }\end{array}$ \\
\hline
\end{tabular}

\section{Conclusões}

As variedades de soja diferem quanto a sensibilidade aos herbicidas metsulfuronmethyl, metsulfuron-methyl + glyphosate, [MSMA + diuron] e [MSMA + diuron] + glyphosate.

Os herbicidas [paraquat + diuron] e 2,4D são eficientes no controle da soja RR.
$\mathrm{O}$ [MSMA + diuron] e [MSMA + diuron] + glyphosate controlou, com eficácia, $\begin{array}{llllll}\text { apenas a variedade } & \mathrm{CD} & 219 & \mathrm{RR}^{\circledR} & \mathrm{e}\end{array}$ metsulfuron-methyl + glyphosate as variedades M-SOY $7908 \mathrm{RR}^{\circledR}, \mathrm{P} 98 \mathrm{Y} 11^{\circledR}, \mathrm{P} 98 \mathrm{R} 31^{\circledR}$, BRS Favorita RR ${ }^{\circledR}$ e CD 219 RR $^{\circledR}$.

A adição de glyphosate não acarretou prejuízos na eficácia dos herbicidas e potencializou a ação do metsulfuron-methyl. 


\section{Referências}

BOND J.A.; WALKER T. Control of volunteer glyphosate-resistant soybean in rice. Weed Technology, v.23, n.2, p.225-230, 2009.

BRADSHAW, L.D. et al. Perspectives on glyphosate resistance. Weed Technology, v.11, n.2, p.189-190, 1997.

BRYSON, C.T.; REDDY, K.N.; MOLIN, W.T. Purple nutsedge (Cyperus rotundus) population dynamic in narrow row transgenic cotton (Gossypium hirsutum) and soybean (Glycine max) rotation. Weed Technology, v.17, n.4, p.805-810, 2003.

CRUZ, C.D. Programa Genes - Estatística Experimental e Matrizes. 1. ed. Viçosa: Editora UFV, 2006. v.1, 285p.

DAN, H.A. et al. Controle Químico de plantas voluntárias de soja Roundup Ready ${ }^{\circledR}$. Revista Brasileira de Herbicidas, v.8, n.3, p.96-101, 2009.

EDENFIELD M.W. et al. Effect of glyphosate and MSMA application timing on weed control, fruiting patterns, and yield in glyphosate-resistant cotton. Weed Technology, v.19, n.2, p.224-230, 2005.

LI, J. et al. Influence of formulation and glyphosate salt on absorption and translocation in three annual weeds. Weed Science, v.53, n.2, p.153-159, 2005.

MENEGATTI, A.L.A.; BARROS, A.L.M. Análise comparativa dos custos de produção entre soja transgênica e convencional: um estudo de caso para o Estado do Mato Grosso do Sul. Revista de Economia e Sociologia Rural, v.45, n.1, p.163-183, 2007.

MOLIN, W.T.; HIRASE, K. Comparison of commercial glyphosate formulations for control of prickly sida, purple nutsedge, morningglory and sicklepod. Weed Biology and Management, v.4, n.3, p.136-141, 2004.
MOLIN, W.T.; HIRASE, K. Effects of surfactants and simulated rainfall on the efficacy of the Engame formulation of glyphosate in johnsongrass, prickly sida and yellow nutsedge. Weed Biology and Management, v.5, n.3, p.123-127, 2005.

MONQUERO, P.A. Plantas transgênicas resistentes aos herbicidas: situação e perspectivas. Bragantia, v.64, n.4, p.517-531, 2005.

PETTER, F.A. et al. Associações entre o herbicida glyphosate e inseticidas na cultura da soja Roundup Ready ${ }^{\circledR}$. Planta Daninha, v.25, n.2, p.389-398, 2007.

PLINE-SRNIC, W. Physiological mechanisms of glyphosate resistance. Weed Technology, v.20, n.2, p.290-300, 2006.

SANTOS, J.B. et al. Ação de herbicidas sobre o crescimento de estirpes de Rhizobium tropici. Planta Daninha, v.24, n.3, p.457-465, 2006a.

SANTOS, J.B. et al. Action of two herbicides on the microbial activity of soil cultivated with common bean (Phaseolus vulgaris) in conventional-till and no-till systems. Weed Research, v.46, n.4, p.284-289, 2006 b.

SANTOS, J.B. et al. Efeito de formulações na absorção e translocação do glyphosate em soja transgênica. Planta Daninha, v.25, n.2, p.381388, 2007.

SANTOS, J.B. et al. Tolerance of Bradyrhizobium strains to glyphosate formulations. Crop Protection, v.24, n.6, p.543-547, 2005.

STOCK, D.; HOLLOWAY, P.J. Possible mechanism for surfactant-induced foliar uptake of agrochemicals. Pesticide Science, v.38, n.23, p.165-177, 1993.

TERASAWA, J.M. et al. Antecipação da colheita na qualidade fisiológica de sementes de soja. Bragantia, v.68, n.3, p.765-773, 2009. 
WEBSTER, E.P.; BRYANT, K.J.; EARNEST, L.D. Weed control and economics in notransgenic and glyphosate-resistant soybean (Glycine max).

Weed Tecnology, v.13, n.4, p.586-593, 1999. 